\title{
Forest-cover monitoring based on high resolution remote sensing image
}

\author{
Jiajie Cui ${ }^{1, a}$, Shiming $\mathrm{Li}^{2, \mathrm{~b}^{*}}$ \\ ${ }^{1}$ China University of Mining \& Technology, Beijing, China \\ ${ }^{2}$ Research Institute of Forest Resource Information Techniques, Beijing, China \\ ${ }^{a}$ cjj4895@163.com, ${ }^{b}$ lism@ifrit.ac.cn
}

\begin{abstract}
Keyword: High resolution; Multi-scale segmentation; KNN; Rules-based; Forest-cover monitoring Abstract: The article put forward a method on forest-cover monitoring based on high resolution remote sensing data of adjacent two year and existing vector data of forest sub-compartment. With the GF- 2 remote sensing image and Rapid-Eye images as the data sources, multi-scale segmentation under the forest land boundary control was conducted, and object of deforestation samples were selected. The object-oriented method of KNN and rules-based were used to classify the image and extract the patches of deforestation. Then we get the deforestation vector diagram of early and later periods. The deforestation patches of two periods were compared and analyzed to achieve the deforestation and recovering vector diagram in a year. Finally, the paper proved the feasibility of the method in forest-cover monitoring application by accuracy analysis.
\end{abstract}

\section{Introduction}

Forest resource is an integral part of the national natural resources and the important material basis of human survival and development. It plays an irreplaceable role of supporting the sustainable development of economic and social well-being. Because the forest resource is renewable, it is always in a dynamic change for a long time. In order to grasp the present situation and changes of forest resources and predict trends well and provide a scientific basis for the development of forestry policy, management policies, production and operation plans, long-term planning and testing the operating results, we need to survey and monitor regularly the continuous change of forest resource ${ }^{[1]}$. In recent years, with the domestic high spatial resolution remote sensing satellites have launched as well as the growing popularity and in-depth of GIS technology in the application of forest investigation and management, Remote Sensing becoming an effective means to monitor change of the forest resources. Its widely-covered and repeat visits exhibit large advantage in monitoring changes in forest resources. It also has been widely used to monitor changes in forests of different regions.

Before the appearance of the high spatial resolution satellite, researchers usually used the coarse or medium resolution satellite data for monitoring forest-cover. Muchoney and Haack ${ }^{[2]}$ used the image difference method to study the change of forest landscape caused by defoliation. Sean P.Healey ${ }^{[3]}$ monitored vegetation change by tasseled cap transformation of Landsat 8 data. Zeng Qingwei ${ }^{[4]}(2008)$ used Landsat TM data to study the forest vegetation changes by the ratio vegetation index under the Imagine ERDAS 9.1 platform. With the launch of the high spatial resolution satellite, the rules-based change detection technology has been developed rapidly. In 2011, the rules-based method was used by Nesrine Chehata ${ }^{[5]}$ on forest disaster detection and they employed mean shift method on segmentation and classification. Zhang Xiaodong ${ }^{[6]}$ used the rules-based method to classify and compare the results to realize the change detection assisted with GIS data, and he achieved good results. Qin Yong ${ }^{[7]}$ et al. used the method of support vector machine to detect the land-use change with high resolution remote sensing image. The experimental results demonstrated the feasibility of the Quickbird image in land-use change detection in the 
county and the small area. Nowadays, the single use of medium and low resolution remote sensing image in forest-cover monitoring has been unable to meet the requirements of production practice. Such as GF-2, SPOT5, GF-1, QuickBird, Rapid-Eye and other high-resolution remote sensing of optical image is increasingly applied to forest-cover monitoring.

\section{Study Area and Data}

Study Area. According to the existing data and auxiliary data, a pilot area of Bobai, Yulin City of Guangxi Zhuang Autonomous Region in South central China is chosen as the research area, which is located at approximately $21^{\circ} 55^{\prime} \mathrm{N}$ in latitude, and $109^{\circ} 54^{\prime} \mathrm{E}$ in longitude. The terrain is mainly mountainous, and is Subtropical monsoon climate of South Asia, with intense sunlight and moderate rainfall. So the vegetation cover rate is higher. The main tree species is fast-growing Eucalyptus, which is a rotation cycle of 4 years or so. Thus the forest-cover changed quickly. Fig. 1 is the remote sensing image of the early and late stage of the experimental area.

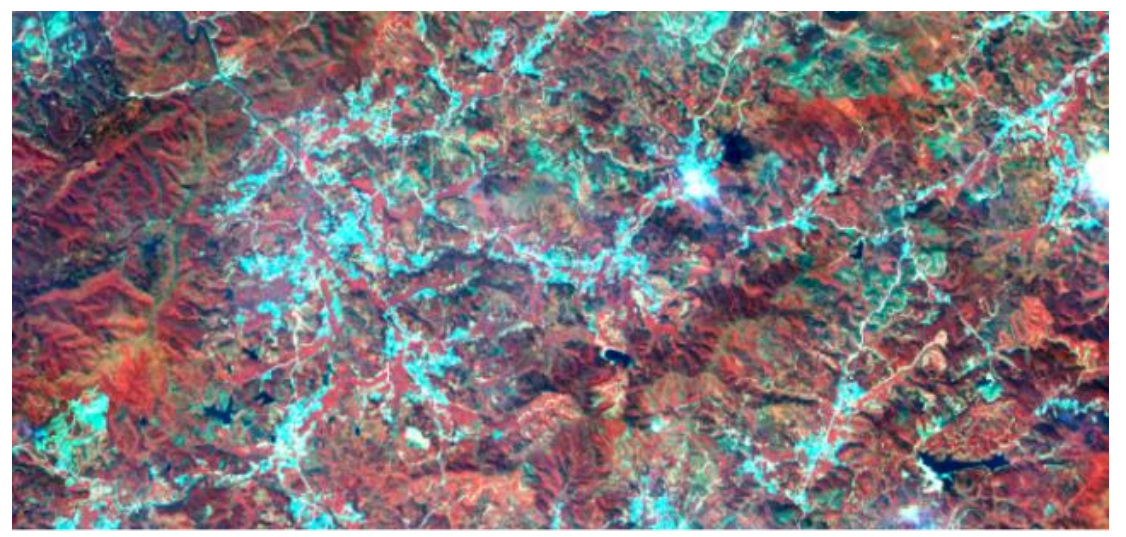

(a) Rapid-Eye Remote sensing image

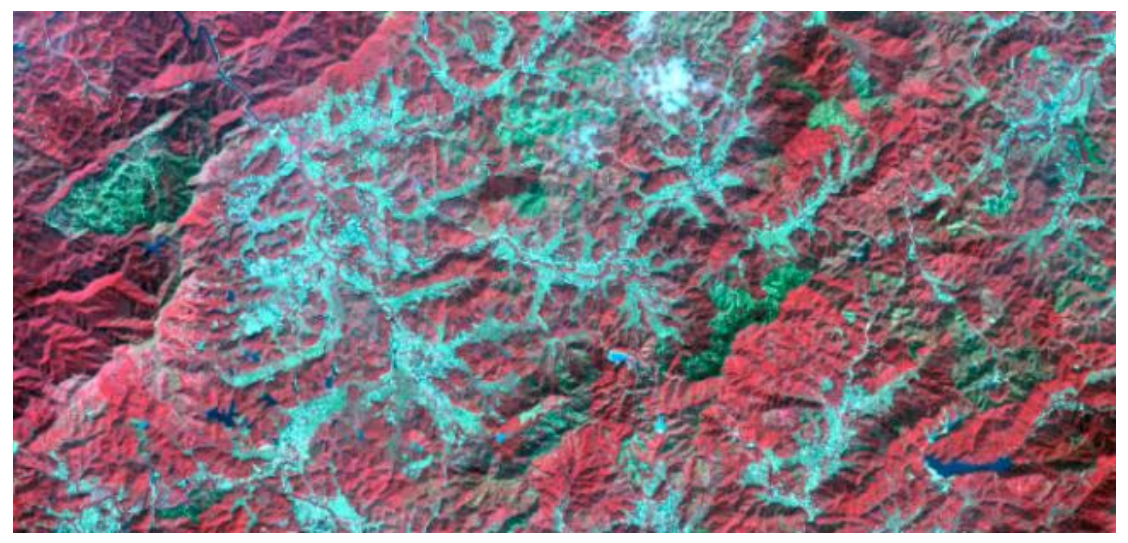

(b) GF-2 Remote sensing image

Fig 1. The remote sensing image of the early and late stage of the experimental area

Data. The paper employed the following experimental data: (1) The domestic high resolution remote sensing image of No.2 (GF-2). Its spatial resolution is $4 \mathrm{~m}$, including four bands: blue, green, red, and near infrared. Acquisition time is 2014-11-16. (2) Rapid-Eye satellite image with geometric correction. Acquisition time is 2013-09-18. Its spatial resolution is 5m. (3) DEM data of the experimental area. Its spatial resolution is $30 \mathrm{~m}$. Due to the terrain of experimental area is mainly mountain, and the difference of topography is relatively large. Considering to using a digital elevation model (DEM) to achieve high-precision image correction. (4) The sub-compartment vector data of Forest Resource Inventory, which is assisted to analyze and extract forest area. 


\section{Data Preprocessing}

In order to eliminate geometric distortion of the image, we used ERDAS software to process the GF-2 data for geometric correction. During the correction process, the RPC parameter, which is provided by the image dataset and DEM data of the region were employed to correct the GF-2 image, referenced the Rapid-Eye satellite image. The calibration model of polynomial and the resampling method of cubic convolution was selected. We Selected 30 control points, which were evenly distributed to insure the registration accuracy of the two images within a pixel.

\section{Methodology}

The experiment used eCognition software to monitor changes, which is an intelligent analysis software developed by German Definiens company. Firstly, two phase images were segmented with proper scale. Secondly, they were classified to deforestation and non-deforestation individually through different methods. Finally, analyzed the results of classification to achieve the change situation of the forest.

\section{Image segmentation}

Segmentation under the control of the forest Vector boundary. Since the sub-compartment vector data of Forest Resource Inventory is cracked, we merged adjacent vector plaque according to the code of same class by the fusion function (dissolve) of ArcGIS, forming the bigger polygons of forest class, namely forest vector boundary. Under the control of forest vector boundary, we obtained a consistent range to the forest vector boundary through image segmentation, which is realized by the chessboard segmentation algorithm of eCognition software. As the image pixels are not involved in computing in the chessboard segmentation algorithm in case of the participation of the vector layer. The operational efficiency of the algorithm is high. Therefore, under the control of the vector boundary we used the chessboard segmentation algorithm. The chessboard size parameter is set larger than the maximum pixels number of the image, aimed at obtaining a consistent image object to the forest vector boundary. Figure 2 shows the image objects by chessboard segmentation, and you can see the image object's boundary is exactly same to the forest thematic boundaries.

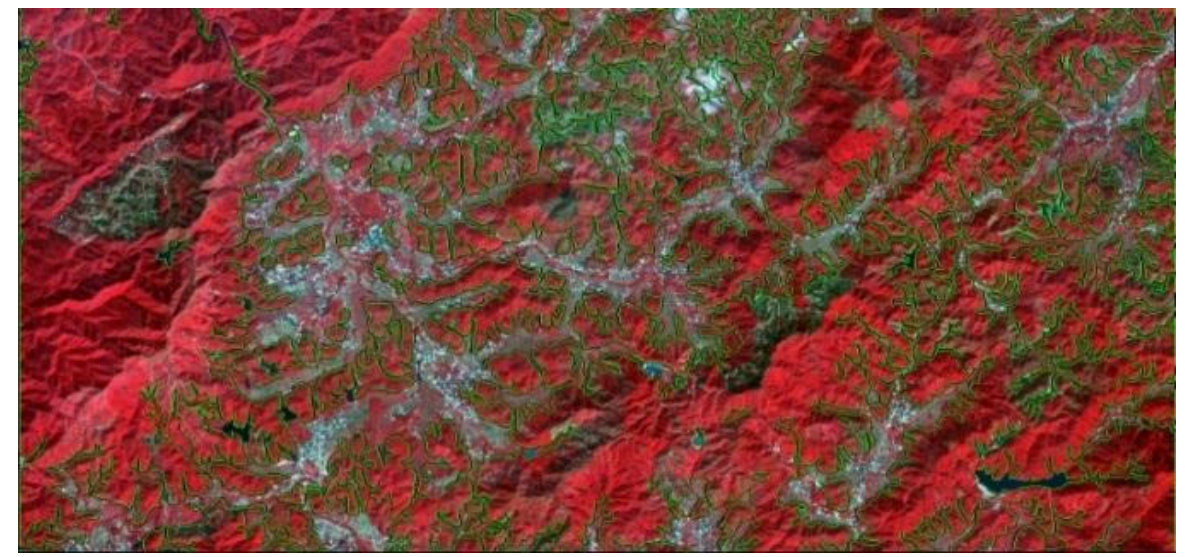

Figure 2 The obtained image objects by chessboard segmenta -tion algorithm.

Multi-resolution segmentation based on forest object. The main purpose of the image segmentation is to merge the single image pixels or sub-objects to form a larger image object based on criteria over the spectral and spatial properties. The multi-resolution segmentation algorithm used in the eCognition software is an iterative process of local optimization based on the average homogeneity of the created objects. The measure of the homogeneity includes the spectral and spatial components. The spectral homogeneity is defined as the average values of the generated objects. Meanwhile, the spatial homogeneity is formed by the two shape features: smoothness (the ratio between the perimeter of the object and the perimeter of the minimum boundary rectangle) and 
compactness (the ratio between the perimeter of the segment and the square root of its area) ${ }^{[8]}$. Thus, the segmented objects, in comparison with single pixels, have richer spectral information, such as object's mean values per band, median values, minimum and maximum values, mean ratios, variance, and so on. However, the merging process can lead to over or under-segmentation. Hence, the optimal scale parameter must be taken into consideration to obtain the best segmentation result.

Scale parameter is an important value, which is the criteria to determine whether a pixel merged into adjacent image objects. It determines the size of segmented image objects and the accuracy of extraction. In order to obtain effective segmentation of image objects, here we select three segmentation scale to test: 100,120,150. Due to the different resolution of the two phase image, we employed different segmentation scale. After several tests, image segmentation parameters were shown in the following table, which can obtain ideal segmentation result.

\begin{tabular}{|l|c|c|c|}
\multicolumn{2}{c|}{ Table 1 } \\
$\begin{array}{l}\text { Shape } \\
\text { weight }\end{array}$ & $\begin{array}{l}\text { Shape } \\
\text { Weight }\end{array}$ & Compactness & Scale \\
\hline Rapid-Eye & 0.1 & 0.5 & 120 \\
\hline GF-2 & 0.2 & 0.5 & 100 \\
\hline GF-2 & 0.2 & 0.5 & 100 \\
\hline
\end{tabular}

Image classification. Firstly, the method of K-Nearest Neighbor (KNN) was used to classify the forest object of early images (Rapid-Eye), which is similar to the traditional classification method of supervised classification by selecting samples to classify. The principle is to calculate the feature of selected samples, taking this feature as the center. Then make the "difference" between features of sample and features used to classify in unclassified object as the distance. The closest image object to the sample category will be assigned to the sample class. The advantage of this method is simple, intuitive and fast.

Then we used the rules-based method to classify the GF-2 image. Since the same band of different objects absorb and reflect sunlight on various degree, and the ability of same object in different wavelengths to absorb and reflect sunlight are different. According to this principle, we sampled the spectra of deforestation samples to obtain the spectrum mean value and texture feature value. When the forest was deforested, the vegetation index decreased, and we selected the vegetation index (NDVI) as a threshold condition. We discovered that deforestation samples in the red band were significantly different to the no-deforestation through analys is of object features. So we selected the vegetation index combined with the mean value of red band to extract the deforestation area. The deforestation area extraction rules of GF-2 image was: NDVI $<0.188$, mean red $>200.5$. Figure 3 shows the classification results of two images.

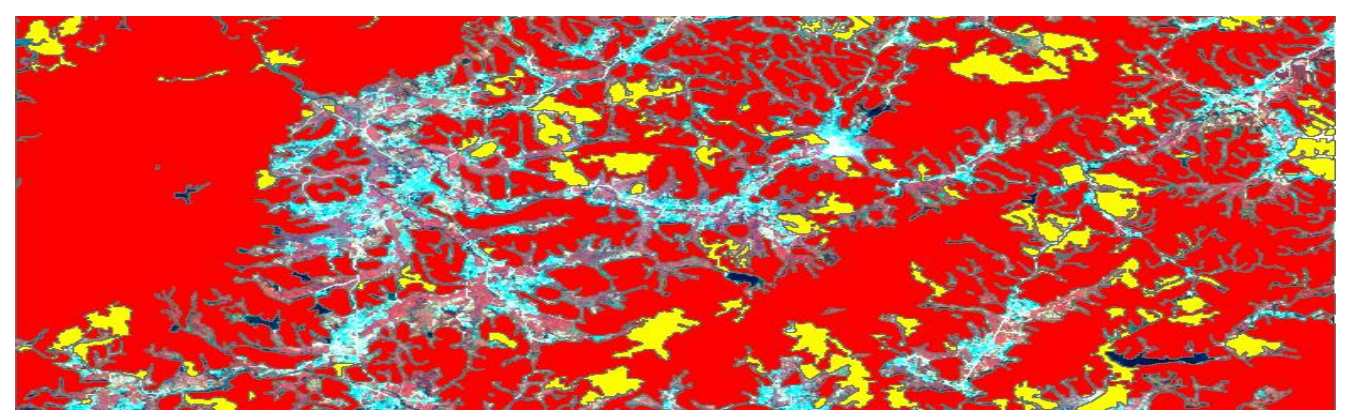

(1) The Early image (Rapid-Eye) forest classification map (yellow patches are deforestation area and red patches are forest) 


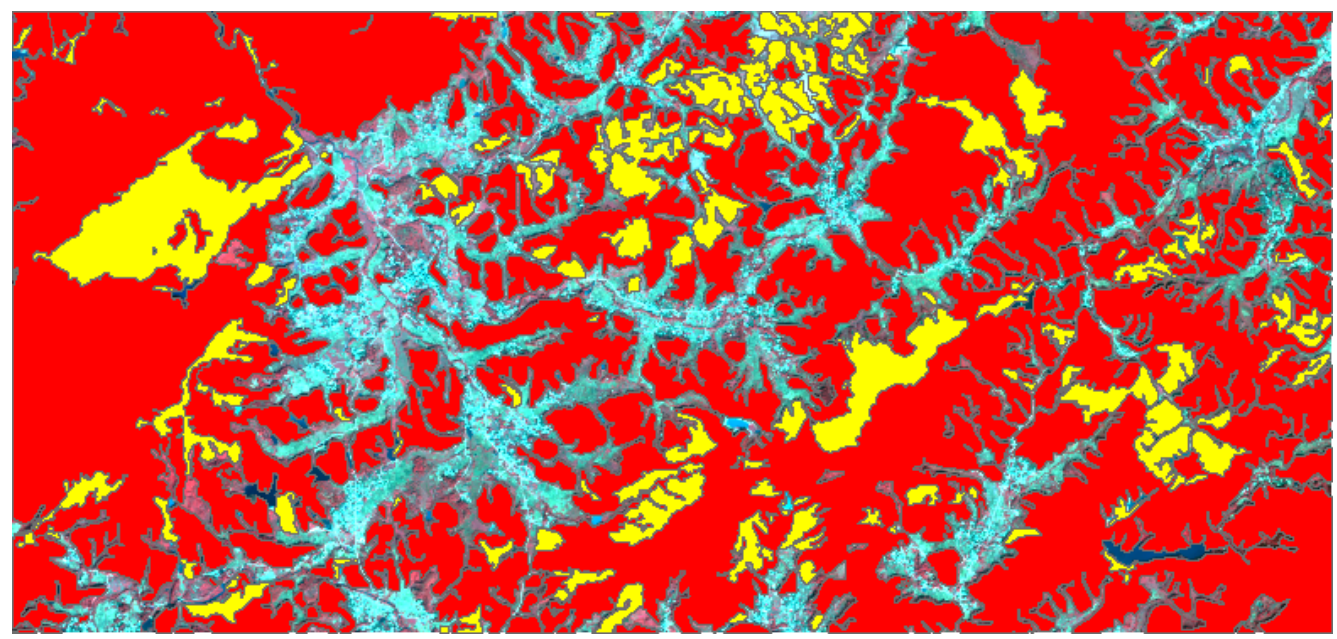

(2) The later image (GF-2) forest classification map (yellow patches are deforestation area and red patches are forest)

Figure 3. The classification results of two phase image

Change detection. We analyzed the overlay of the two vector classification results in ArcGIS software, and obtained the deforestation and restoration change map of the experimental zone. The result was shown below:

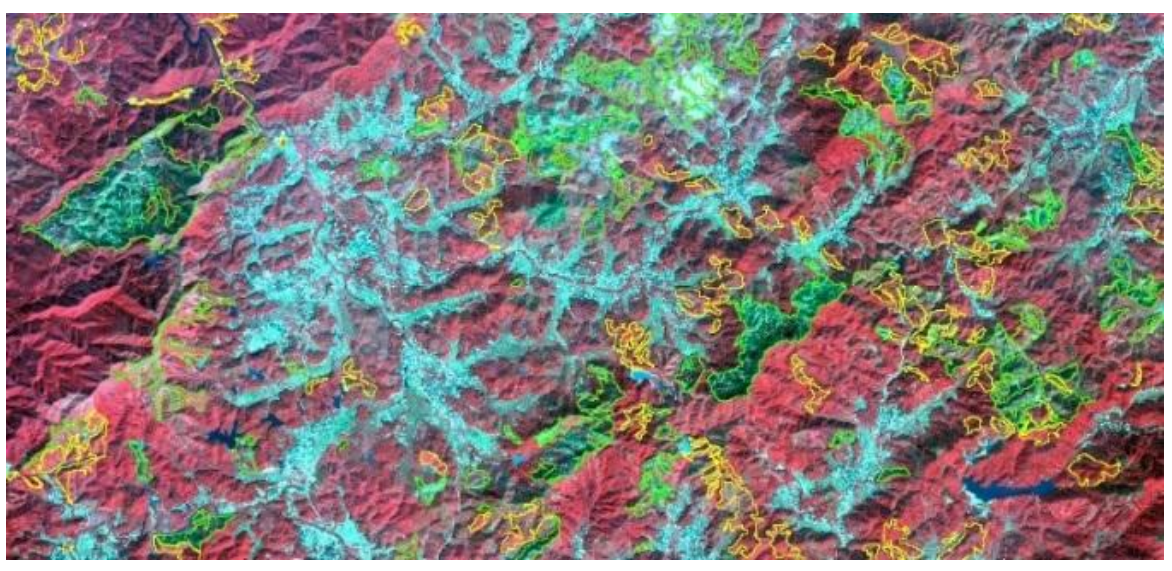

Figure 4. The deforestation and restoration change map (the Green patches are deforestation area and the yellow patches are restoration area)

\section{Conclusions}

In the experimental area, we selected randomly 100 points which are visual changes as a verification to evaluate the accuracy of detected change polygons. The results are as follows:

Table 2 The accuracy of detected change

\begin{tabular}{|l|l|l|l|l|l|l|}
\hline Type & $\begin{array}{l}\text { Sample } \\
\text { NO. }\end{array}$ & $\begin{array}{l}\text { Correct } \\
\text { NO. }\end{array}$ & $\begin{array}{l}\text { Error } \\
\text { NO. }\end{array}$ & $\begin{array}{l}\text { Missed } \\
\text { NO. }\end{array}$ & $\begin{array}{l}\text { Error } \\
\text { rate }\end{array}$ & $\begin{array}{l}\text { Missed } \\
\text { rate }\end{array}$ \\
\hline Change points & 100 & 82 & 7 & 11 & $7 \%$ & $11 \%$ \\
\hline
\end{tabular}

Then, we selected randomly 20 samples to manually digitalize, which is regarded as criterion to evaluate the area of detected object. We called it area overlapping ratio. It is the ratio between the area of overlapping polygon and digitalized samples area. The overlapping polygon is the sharing area of detected change area and digitalized samples. The ratios are as follows. 


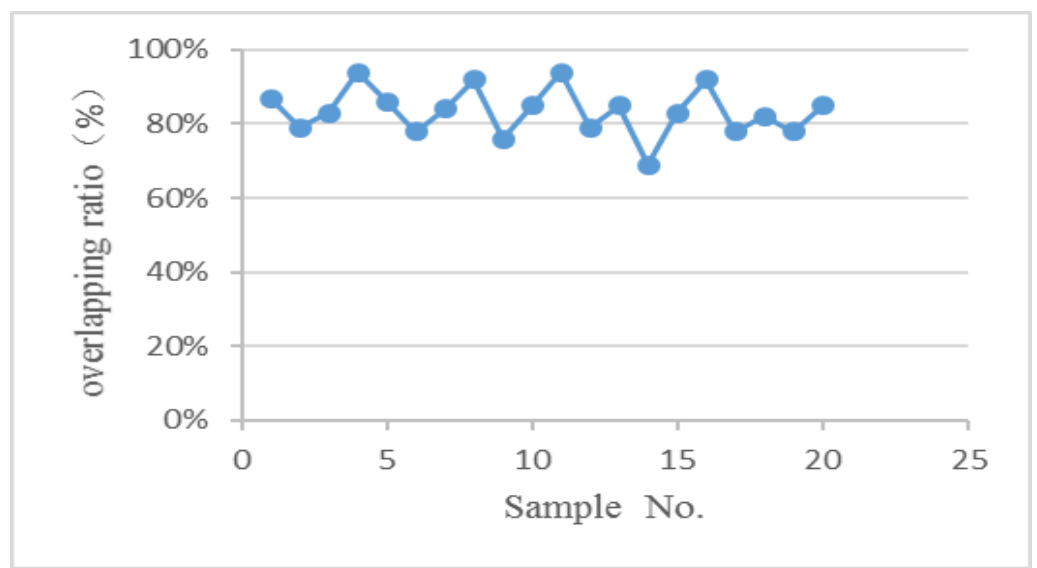

Figure 5. Overlapping ratio of detected change and digitalized samples

Contrasting the overlapping area between detected polygons area and digitalized sample area, the average ratio is $83 \%$, which is within the allowable limits of detection error. It proved the accuracy and feasibility of this approach and meet the accuracy requirements. It can be used to practical applications of annual forest-cover deforestation monitoring.

\section{Acknowledgments}

This research was supported by the National Science and Technology Major Projects of China (the project number is 21-Y30B05-9001-13/15) and the National Natural Science Foundation of China (the project number is 31370635). The authors would like to thank the Research Institute of Forest Resource Information Techniques, Chinese Academy of forestry for providing the satellite and ancillary data used in this study.

\section{References}

[1] M.KOHL. Forest inventory and monitoring. Encyc-lopedia of Forest Science,2004, pp. 403-409.

[2] D.M.Muchoney, B.N.Haack, Detection for monitoring forest defoliation. Photogrammetric Engineering and Remote Sensing,1994, pp.1243-1251.

[3] S.P. Healey, W.B. Cohen, Z.Q.Yang, O.N. Krankina, Comparison of Tasseled Cap-based Landsat data structures for use in forest disturbance detection . Remote Sensing of Environment, 2005, pp.301-310.

[4] Q.W. Zeng, H.Wu, S.Miao. Study on Remote sensed monitoring forest canopy change based on TM data. Remote Sensing Technology and Application, 2009, pp.186-191.

[5] N.Chehata, C.Orny, S.Boukir, D.Guyon. Object-based forest change detection using high resolution satellite images. ISPRS Conference PIA, 2011, pp.49-54.

[6] X.D.Zhang. The Theory and Methods of Change Detection Based on Remotely Sensed Imagery and GIS. Wuhan University.PhD. Dsisertation. (2005)

[7] Y.Qing, W.H.Kong, J.R.Cao, etc. Land Use Change Detection Based on SV- M .Journal of University of Jinan (Science and Technology), 2010, pp. 88-90.

[8] P.Happ, R.Ferreira, C.Bentes, G.Costa, R.Feitosa, In Multi-resolution Segmentation: A Parallel Approach for High Resolution Image Segmentation in Multicore Architectures. In Proceedings of 
the 3rd International Conference on Geographic Object-Based Image Analysis. (2010) 